\title{
CHARGE-DENSITY DOMAINS IN PHOTOEXCITED QUANTUM-WELL STRUCTURES
}

\author{
R. MERLIN, N. MESTRES, A. McKIERNAN \\ Department of Physics, The University of Michigan, Am Arbor, MI 48109, USA \\ J. OH and P.K. BHATTACHARYA \\ Department of Electrical Engineering and Computer Science, The University of Michigan, Ann Arbor, MI 48109, USA
}

Received 20 June 1989; accepted for publication 18 July 1989

\begin{abstract}
We present theoretical and experimental results on the dielectric response of an electron-hole plasma confined to a quantum-well. Hartree calculations reveal large nonlinearities in the screening properties which, when combined with those of the equations: determining the photoexcited plasma density, can lead to bistable behavior and the formation of domains of different carrier densities. Electronic Raman scattering data showing evidence of such domains are discussed.
\end{abstract}

Electric-field effects in semiconductor quantum-wells (QW's) and, in particular, GaAs- $\mathrm{Al}_{x}$ $\mathrm{Ga}_{1-\lambda} \wedge$ s structures have attracted much theoretical and experimental attention in recent years [1-6]. This is motivated in part by some unique features of the Stark effect in confined geometries, and also by possible device applications including, e.g., light-modulators and infrared detectors [1-6]. In this work, we concern ourselves with the capacitive behavior of photoexcited QW's. Using a simple model, we find that the dielectric response of such a system exhibits two markedly different regimes which cross when the external charge and the electron-hole areal densities become equal. Focusing on particular rate conditions, we predict that nonlinearities in the response can lead to bistability associated with domains of different plasma concentrations. These charge-density domains are a single-well phenomenon and, accordingly, they differ significantly from those associated with resonant tunneling [7]. Preliminary Raman data on GaAs- $\mathrm{Al}_{x} \mathrm{Ga}_{1-x}$ As multiple QW's are presented showing evidence of charge-density instabilities.

Consider a quasi-two-dimensional electronhole plasma, generated by a monochromatic pho- ton source of flux $S$ and frequency $\omega_{\mathrm{I}}$, which interacts with an external electric field due to uniformly distributed charges $\pm Q_{c}$. of a parallelplate capacitor. The plasma is confined to a semiconductor QW of thickness $2 L$ and effective-mass potentials $W_{\mathrm{e}, \mathrm{h}}(z)=\Delta_{\mathrm{e}, \mathrm{h}}\left[W_{\mathrm{e}, \mathrm{h}}(z)=0\right]$ for $|z| \geq L$ $[|z| \leq L]$ (the $z$-axis and the field are normal to the layers, and $\Delta_{\mathrm{c}}$ and $\Delta_{\mathrm{h}}$ are the conduction and valence band offsets). In the Hartree approximation, the envelope functions and eigenenergies are of the form $\Phi=\psi_{j}(z) \exp (\boldsymbol{i} \boldsymbol{k} \cdot \boldsymbol{r})$ and $\Lambda=\Lambda_{j}+$ $h^{2}|\boldsymbol{k}|^{2} /\left(2 m_{\mathrm{e} . \mathrm{h}}\right)$ where $j \geq 0$ is the subband index. $\boldsymbol{k}$ is a wavevector parallel to the interfaces and $m_{e}, m_{\mathrm{h}}$ are the electron and hole masses. The Hartree equations for the subband eigenfunctions $\psi_{i}(z)$ are [8]:

$$
\begin{aligned}
& -\left(h^{2} / 2 m\right) \mathrm{d}^{2} \psi_{j} / \mathrm{d} z^{2} \\
& +\left[W(z)-4 \pi q Q_{\mathrm{C}} z /(A \kappa)+q V_{\mathrm{H}}(z)-\Lambda_{j}\right] \psi_{j} \\
& \quad=0 .
\end{aligned}
$$

where $\psi_{j}=\psi_{j, e}\left(\psi_{j, h}\right), \Lambda_{j}=\Lambda_{j, c}\left(\Lambda_{i, h}\right), m=m_{i}$ $\left(m_{\mathrm{h}}\right), W=W_{\mathrm{e}}\left(W_{\mathrm{h}}\right)$ and $q=-|e|(+|e|)$ for electron (hole) states; $\kappa$ is the appropriate dielectric constant of the semiconductor and $A$ is the area of the capacitor plates. The electrostatic 


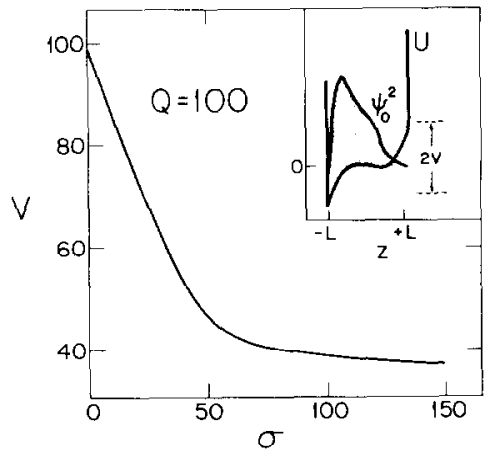

Fig. 1. $V$ versus $\sigma$ for $Q=100$. Inset: diagram of the system showing the total potential $U(z)$ and $\psi_{0}^{2}(z)$ for $Q=\sigma=100$. $\psi_{0}(z)$ and $\psi_{0}(-z)$ are the lowest self-consistent electron and hole eigenstates, respectively.

mean-field potential $V_{\mathrm{H}}(z)$ satisfies the Poisson's equation [8]

$$
\frac{\mathrm{d}^{2} V_{\mathrm{H}}}{\mathrm{d} z^{2}}=\frac{4 \pi|e|}{\kappa} \sum_{j}\left[n_{j, \mathrm{e}} \psi_{j, \mathrm{e}}^{2}(z)-n_{j, \mathrm{~h}} \psi_{j, \mathrm{~h}}^{2}(z)\right],
$$

where $n_{j \mathrm{e}}\left(n_{j \mathrm{~h}}\right)$ is the electron (hole) steady-state concentration in the $j$ th subband. The nonlinear eigenvalue problem, eqs. (1) and (2), can be solved using standard numerical methods [8]. Its solutions $\left\{\psi_{j, \mathrm{e}}, \Lambda_{j, \mathrm{e}}\right\}$ and $\left\{\psi_{j, \mathrm{~h}}, \Lambda_{j, \mathrm{~h}}\right\}$ are expressed in terms of the input parameters $Q_{\mathrm{C}}$ and the populations $\left\{n_{j . \mathrm{e}}\right\}$ and $\left\{n_{j . \mathrm{h}}\right\}$. In photoexcitation experiments, however, the subband concentrations are not determined a priori: the recombination, scattering and tunneling times entering in the rate equations depend, in turn, on $\left\{\psi_{j, \mathrm{e}}, \Lambda_{j, \mathrm{e}}\right\}$ and $\left\{\psi_{j, \mathrm{~h}}, \Lambda_{j, \mathrm{~h}}\right\}$. This leads to a system of coupled nonlinear equations which can exhibit multiple solutions for given values of $S, \omega_{\mathrm{L}}$ and $Q_{\mathrm{C}}$.

In fig. 1, we show the calculated potential drop across the well as a function of plasma density at a constant external charge [9]. The results are for a neutral plasma of electrons and holes of equal masses confined to a slab, i.e., $m_{\mathrm{e}}=m_{\mathrm{h}}=m_{0}$ and $\Delta_{i}=\Delta_{\mathrm{h}}=\infty$. In addition, it is assumed that only the lowest subbands are occupied $\left(n_{j, \mathrm{e}}=n_{j . \mathrm{h}}=\right.$ $\left.n_{0} \delta_{j 0}\right)$. This limiting case contains the necessary ingredients for bistable behavior while considerably simplifying the calculations. $V$ is half the potential difference in units of $\lambda /|e|$, where $\lambda=$ $\hbar^{2} / 2 m_{0} L^{2}$. The nondimensional carrier density $(\sigma)$ and external charge $(Q)$ are $n_{0}$ and $Q_{C}$ in units of $\kappa \lambda / 2 \pi e^{2} L$ and $4 \pi e L / \kappa \lambda A$, respectively. At low carrier densities, $V$ varies linearly with $\sigma$. With increasing $\sigma, V$ crosses over (at $\sigma \approx Q / 2$, i.e., $Q_{\mathrm{C}}=e n_{0} A$ ) to a regime showing a much weaker dependence on the concentration. Using WKB methods, it can be proved [9] that $V(Q, \sigma)$ $\propto \sigma^{-1 / 4}$ for $\sigma \rightarrow \infty$. The two regimes $(Q / 2 \ll \sigma$ and $Q / 2 \gg \sigma$ ) characterizing the screening properties of the plasma determine the nonlinear capacitive behavior of the QW under photoexcitation. Bistability, as discussed below, corresponds to situations where high- $\sigma$ and low- $\sigma$ states can coexist for a given set of parameters $S, \omega_{\mathrm{L}}$ and $Q$.

Electric fields produce a red-shift of the $Q W$ absorption edge $\Theta_{\mathrm{W}}[1,2]$. Therefore, if $\Theta_{\mathrm{W}}(Q=0)$ is slightly larger than $\hbar \omega_{\mathrm{L}}$, the condition for creating a plasma $\left(\hbar \omega_{L} \geq \Theta_{\mathrm{W}}\right)$ will eventually be met at a certain value of the field. The photogenerated plasma itself complicates this picture. Its effect on $\Theta_{\mathrm{W}}$ opposes that of $Q$ and, provided $S$ is sufficiently large, there will be a strong competition between the field and the plasma to define the state of the system. As discussed in the following example, this charge-induced feedback can lead to bistability. Like in the previous paragraph, we consider a situation where the photogenerated carriers populate predominantly the lowest subband. In addition, the time evolution of the electron (or hole) density is assumed to obey the rate equation $\tau_{\mathrm{R}} \mathrm{d} \sigma / \mathrm{d} \iota=G-\sigma$, where $\tau_{\mathrm{R}}$ is the radiative recombination lifetime and $G=4 \pi e^{2} L^{2} S \tau_{\mathrm{R}} \alpha /$ ( $\left.\hbar \omega_{L} \kappa \lambda\right)$ is the generation rate ( $\alpha$ is the absorption coefficient at $\left.\hbar \omega_{\mathrm{L}}\right)$. The dependence of $G$ on the state of the plasma involves the product $\tau_{\mathrm{R}} \alpha$. Using the envelope-function approximation and ignoring band-filling effects, one finds $G \propto$ $\tau_{\mathrm{R}}^{0} \alpha_{0}(h \Omega)$. Here, $\alpha_{0}(h \Omega)$ and $\tau_{\mathrm{R}}^{0}$ are the absorption coefficient and the radiative lifetime at zerofield, and $\hbar \Omega=\left[\hbar \omega_{\mathrm{L}}-\Theta_{\mathrm{W}}(Q, \sigma)\right]$ (i.e., $Q$ and $\sigma$ enter only through the shift of the absorption edge). In the steady-state, $G=\sigma$. Given $\left\{S, \omega_{\mathrm{L}}, Q\right\}$ and assuming absorption by excitons, i.e., $\alpha_{0} \propto\left[(\hbar \Omega)^{2}+\Gamma^{2}\right]^{-1}$, the latter equation exhibits a range of multiple solutions. This is shown in fig. 2 (values of the parameters used are indicated in the caption). The lowest branch corre- 
sponds to $h \omega_{\mathrm{L}} \leq \Theta_{\mathrm{w}}$ and terminates at the resonance $(\Omega-0)$ where $\sigma$ is largest while, for the upper branch, $\sigma \ll V$, i.e., $V \approx Q$. The light-controlled capacitor operates as a switch: stability against $\sigma$-fluctuations imposes the condition $\partial G / \partial \sigma<1$ leading to an unstable middle branch.

The Raman results shown in fig. 3 reveal evidence of bistable behavior. The data were obtained on a sample consisting of forty periods of $212 \AA \mathrm{GaAs} / 160 \AA \mathrm{Al}_{0.7} \mathrm{Ga}_{0.3}$ As with $\omega_{\mathrm{L}}$ in the vicinity of the $E_{0}+\Delta_{0}$ resonance of GaAs. The features indicated by arrows correspond to coupled intersubband-LO (longitudinal optical) phonon excitations [10]. From their positions, values for the bare intersubband energy and the carrier density in the wells can be inferred [10]. The observation of two sets of coupled modes in the spectra at -5.2 and $-6.0 \mathrm{~V}$ is ascribed to bistability associated with resonant photoexcitation. The plasma densities are in the range $8.0 \times 10^{10}$ $1.5 \times 10^{11} \mathrm{~cm}^{-2}$. Experiments at slightly lower power densities, i.e., lower carrier concentrations, show a single domain in the whole voltage-range. This indicates that the domains relate to the photoexcitation process and, furthermore, that the instability is not due to sequential resonant tunneling [7] (the measured positions of the intersubband peaks and the photoinduced $I-V$ response do not support the latter possibility either). The

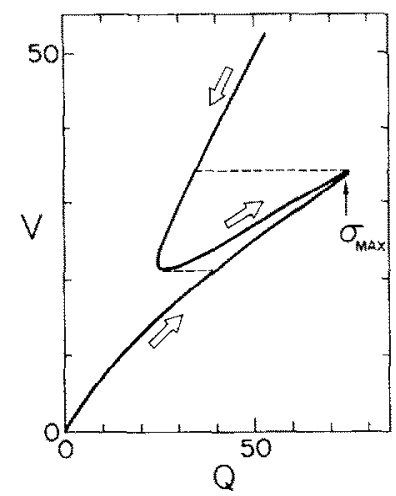

Fig. 2. $V$ versus $Q$ for the case of resonant photoexcitation. Arrows indicate the direction of increasing $\sigma$ and dashed lines show the $V$-range of bistability. The calculations are for $G=$ $30.0\left[\left(\hbar \omega_{1}-\Theta_{W}\right)^{2} / \lambda^{2}+(\Gamma / \lambda)^{2}\right]^{-1}$ with $\hbar \omega_{\mathrm{L}}=\Delta_{\mathrm{G}}$ and $\Gamma / \lambda$ $=1.5 . \Delta_{C}$ is the bulk-semiconductor gap.

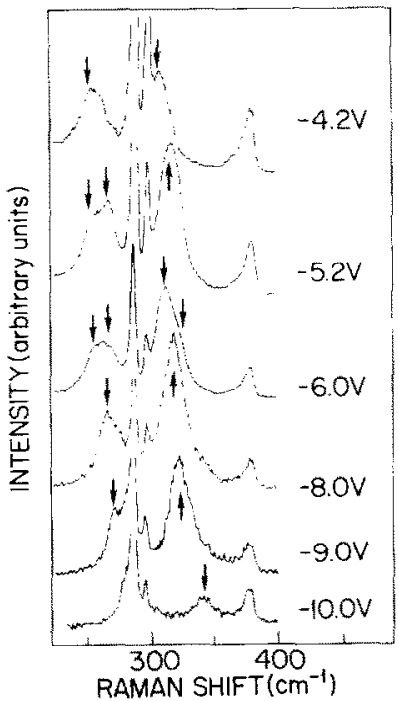

Fig. 3. Raman spectra of the multiple QW structure described in the text at $T=4 \mathrm{~K}$. Arrows denote coupled intersubband-LO GaAs modes. Additional features are LO phonons of the alloy and buffer layers. The scattering geometry is $2\left(x^{\prime}, x^{\prime}\right) z$ and $h \omega_{1}=1.89 \mathrm{eV}$.

model calculation in fig. 2 qualitatively accounts for the experimental findings. However, the observed dependence of the concentration on the voltage does not completely agree with this interpretation.

This work was supported in part by the US Army Research Office under the contract No. DAAL-03-89-K-0047 and by the URI program contract No. DAAL-03-87-K0007.

\section{References}

[1] G. Bastard, E.E. Mendez, L.L. Chang and L. Esaki, Phys. Rev. B 28 (1983) 3241

[2] D.A.B. Miller, D.S. Chemla. T.C. Damen. A.C. Gossard. W. Wiegmann, T.H. Wood and C.A. Burrus, Phys. Rev. Lett. 53 (1984) 2173.

[3] H.-J. Polland, L. Schultheis, I. Kuhl, E.O. Gobel and C.W. Tu, Phys. Rev. Lett. 55 (1985) 2610.

[4] L. Viña, R.T. Collins, E.E. Mendez and W.I. Wang. Phys. Rev. B 33 (1986) 5939

[5] K. Bajema, R. Merlin, F.-Y. Juang, S.-C. Hong, J. Singh and P.K. Bhattacharya, Phys. Rev. B 36 (1987) 1300. 
[6] D. Fröhlich, R. Wille, W. Schlapp and G. Weimann, Phys. Rev. Lett. 59 (1987) 1748.

[7] L. Esaki and L.L. Chang, Phys. Rev. Lett. 33 (1974) 495.

[8] See, e.g., T. Ando, A.B. Fowler and F. Stern, Rev. Mod Phys. 54 (1982) 437, and references therein.
[9] R. Merlin and D.A. Kessler, unpublished.

[10] See, e.g., A. Pinczuk and G. Abstreiter, in: Light Scattering in Solids V, Vol. 66 of Topics in Applied Physics, Ed. M. Cardona and G. Güntherodt (Springer, Berlin, 1989) ch. 4 . 